\title{
Time Variant-Peer Node Heterogeneous Peer to Peer Networks using Dynamic Warping Algorithm
}

\author{
P. Murugesan \\ A. Shanmugam \\ Principal \\ Assistant Professor (SG) \\ Department of CA , BIT, Sathyamangalam-638401 \\ Tamil Nadu, India
}

V.S. Prakash

Assistant Professor

\begin{abstract}
In the current internet scenario more virtual servers and mirror servers are utilized to maintain load balance of the heterogeneous peer to peer networks. One of the most current existing works developed a state balancing system based on probability distribution of peer abilities, loads of virtual servers, and incomplete data of global peers. On the other hand state balancing method recognizing peer node capacities are difficult a) as the demand and weight of the peer differ time to time which requires to be addressed b) In account of this, the peer node persistence needs to be managed with its CPU cycles for processing the client demands c) Load diversion of peer neighbors supply to the difficulty of corresponding peer nodes on serving their vital demand d) At the lower dimension of load balancing, various data format increases the processing time of the peer servers.

To overcome the issues, we plan to build Time Variant-Peer Node Heterogeneous Data Processing Scheme for efficient load balancing in distributed heterogeneous peer networks. Peer node time variant capacity is measured using Dynamic Time Warping (DTW) algorithm to evaluate the magnitude of load-demand balance factors of peer servers. With the resultant load-demand factor obtained from DTW, peer server processing cycle requirements are identified using Duty Cycle Data Appropriation (DCDA) Technique. Load diversions are made to peer server with Node Selection Strategy based on DCDA rank representation. The heterogeneous data nature of the demand requisite by the peer servers is implicitly identified by Apriori of Data Format Load Levels are matched to current data format demand of respective nodes and its effect on load balancing the peer servers are calculated. Experimental performances are evaluated with the Heterogeneous peer networks data extracted from the large internet service providers. In addition simulations are carried out to show the effectiveness of our work with bench mark data sets from UCI Repository.
\end{abstract}

Keywords

Heterogeneous P2P, DTW, DCDA.

\section{INTRODUCTION}

PEER-TO-PEER (P2P) networking is a promising method for next generation network applications. P2P networks are application-level networks constructed on the last part of the systems, which give message routing and delivery. Popular $\mathrm{P} 2 \mathrm{P}$ applications comprise dispersed computing, media streaming, file sharing, and rely on their P2P network framework for information searches, message routing, and/or content delivery. P2P network frameworks are the building blocks in the design and implementation of P2P applications. Probable P2P sub nodes are supported on distributed hash tables or DHTs for short. Instances of DHTs are Chord and Pastry.

Take part peers in a DHT can horde diverse numbers of virtual servers, thus taking advantage of peer heterogeneity. Assign $\mathrm{S}$ is the set of virtual servers, and $\mathrm{N}$ is the set of peers taking part in the system. By reallocating virtual servers in $\mathrm{S}$ to peers in $\mathrm{N}$, recommend that each peer $\mathrm{i}$ belongs to $\mathrm{N}$ contract with the load balancing problem by minimizing the following [1]:

$$
\left|\Sigma \mathrm{s}_{\varepsilon s i} \mathrm{Ls} \quad / \mathrm{C}_{\mathrm{i}}-\mathrm{A}\right| \ldots \ldots \ldots \ldots \ldots \ldots \ldots \ldots(1.1)
$$

Where $S_{i} \in S$ represents the set of virtual servers allocated to peer $\mathrm{i}$

$$
\begin{aligned}
& S_{v} \text { is the load value of a virtual server } s \in S_{i} \text {; } \\
& C_{i} \text { is the capacity value of peer } i \text {; } \\
& A \text { is the expected load value per unit capacity [1] }
\end{aligned}
$$

The main process of the heterogeneous peer networks is that the reallocation of an $\mathrm{S}$ from a source node to a destination node can be just completed by creating the leave and link operations presented by a typical DHT. Then, Ls at a particular interval of time is the sum of the loads of the objects (data items) stored in $\mathrm{v}$ at that time. Possible metrics for measuring the load of an object may include the storage size of the object, the mean bandwidth required for serving the object, and so on. After that, $\mathrm{Ci}$ characterize the highest load that node $i$ is capable to hold, which signify the processor speed, accessible disk space, and the bandwidth of peer $i$. The balancing load factor of the peer nodes which is taking part in the system is disturbed with the minimization of the system wide performance metric-movement cost—as much as possible.

The existing load balancing algorithms that activate in a dispersed behavior and intend at energetic and heterogeneous DHTs which comprise each node, $\mathrm{k}$, naturally identifies a target load, Tk, and Tk $<\mathrm{Ck}$. Beyond Tk, $\mathrm{k}$ will allow virtual servers (S), such that the entire load of the virtual servers (S) is no more than Tk. Apparently, the perfect setting for Tk is $\mathrm{A}^{*} \mathrm{Ck}$, that is, $\mathrm{k}$ is estimated to supervise the load proportional to its capacity. The existing algorithms for predicting the peer nodes capacity on the network is not an efficient to use.

\section{LITERATURE REVIEW}

Many algorithms have been proposed earlier for the load balancing approach used in homogeneous environment. For heterogeneous environment, Hung-Chang Hsiao, Hao Liao 2011 presented a load balancing approach for peer to peer systems. They used reallocation algorithm and distribution algorithm for a heterogeneous peer networks. A novel load balancing algorithm is used here to improve the load balancing factor among the networks. 
Y. Zhu and Y. Hu 2005 presented a technique for a P2P network used distributed hash table for an efficient ProximityAware Load Balancing approach. But the progress is not an efficient for heterogeneous peer networks. There is a chance for getting server problem, for that, C. Chen and K.-C. Tsai 2008 presented a technique for Server Reassignment Problem using load balancing approach in Structured P2P Systems. Y. Zhu 2009 also developed a technique for Load Balancing in Structured P2P Systems.

Wang Wei, Payam Barnaghi ET.AL 2010 developed a probabilistic technique for improving the $\mathrm{P} 2 \mathrm{P}$ network and it can be applied in learning ontologies. S.M. Ross 2007 presented a probabilistic technique for $\mathrm{P} 2 \mathrm{P}$ network and analyzed the process of probability of different types of system in the network. Yuzhe Tang, Shuigeng Zhou ET. AL 2010 presented a LIGHT technique for DHT using a QueryEfficient Yet Low-Maintenance Indexing Scheme.

Prashant Dewan and Partha Dasgupta 2010 developed a technique for P2P Reputation Management Using Distributed Identities and Decentralized Recommendation Chains. S. Idreos, E. Liarou et. Al., 2008 proposed an approach for Distributed Hash Tables which could be used under Continuous Multi-Way Joins. Finally, to improve the heterogeneous P2P network, there we are going to implement a allocation and dynamic warping algorithm to evaluate the load balance factor of the peer nodes participating in the network with virtual servers.

\section{TIME VARIANT-PEER NODE HETEROGENEOUS P2P NETWORKS USING DYNAMIC WARPING ALGORITHM}

\subsection{Reallocation algorithm of virtual server to peer node}

In this paper, we imagine that the total hash space offered by a DHT is $[0,1]$, and each virtual server in the DHT has a distinctive ID chosen separately and consistently at arbitrary from the space $[0,1]$. Let $\mathrm{P}$ be the set of participating peers, and $\mathrm{S}$ be the set of virtual servers taken by the peers in $\mathrm{P}$ in the DHT. Denote the set of virtual servers in peer i by Si. Each peer $\mathrm{i}$ belongs to $\mathrm{P}$ determines the load, which is indicated by $\mathrm{Ti}$, that it should recognize, where $\mathrm{Ti}=\sim \mathrm{A} * \mathrm{Ci}$ $+\epsilon, \sim A$ is an estimation for the expected load per unit capacity,[1] i.e.

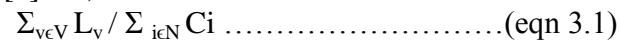

Where $\epsilon$ is pre-defined system parameter. If the present overall load of $i$ is greater than Ti (i.e., $i$ is overloaded), then $i$ drifts its $\mathrm{S}$ to other peers $\mathrm{i}$. Or else it does nothing but remains to accept the drifted virtual servers $S$. If peer $i$ is overloaded, i choose virtual servers $S$ for relocation, such that 1) i turns into under loaded, and 2) the total movement cost, $\mathrm{MC}$, is minimized due to the reallocation. If peer $\mathrm{i}$ is an under loaded, then $\mathrm{i}$ is demanded to accept a drifted virtual server $\mathrm{Si}$, and $i$ admits such a virtual server if the added load due to the virtual server will not overload itself; otherwise, i rejects such virtual server.

Algorithm (Reallocation (i)), [1] which given as follows

illustrates our idea.

Input: $\mathrm{P}, \mathrm{S}, \mathrm{i}, \mathrm{T}$

Output: allocation of $S_{i}$ to $P_{i}$

Switch (Load (i)) do

Case $>\mathrm{T}_{\mathrm{i}}$

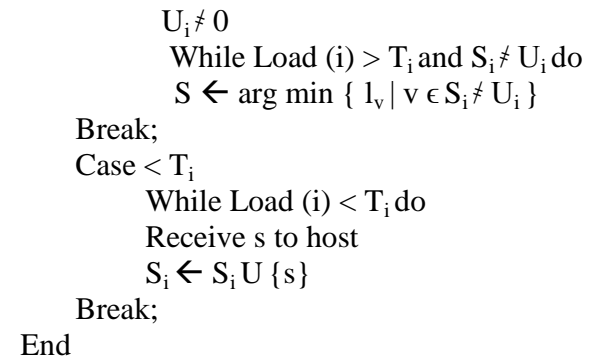

But we don't know that how long the peer node will be in that P2P network. Since there is a chance of peer node to move out of the network, it is necessary to denote the time of the peer node i.e., liveliness of the peer node in the network, To monitor the time of the peer node in the heterogeneous P2P network, the Dynamic Time Warping algorithm is used. The architecture diagram is shown in fig 3.1 .

\subsection{Dynamic Time Warping Algorithm (DTWA)}

The DTWA is used to evaluate the dynamic time variant capacity of peer node to evaluate the magnitude of load-demand balance factors of peer servers.

The DWA for evaluating the time variant capacity of peer node is described below:

Int DTWA time variant capacity $(\mathrm{C}(\mathrm{p} 1), \mathrm{C}(\mathrm{p} 2) \ldots . \mathrm{C}$ (pn), int w)

\{

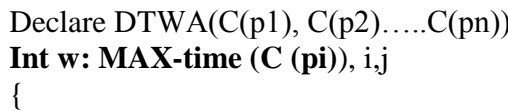

For $\mathrm{i}=1$ to $\mathrm{n}$

End For

Evaluate $\mathrm{w}$ for $\mathrm{C}(\mathrm{pi})$

For $\mathrm{j}=1$ to $n$

Do End Do

End For \}

\section{\} Algorithm1}

The DTWA will efficiently identify the liveliness of the peer node in the network which may vary in time or speed. So the peer node, before share its virtual servers with other node, it will check first the DT (Dynamic Time) of the neighbor peer node using DTWA in the heterogeneous P2P network. After analyzing it, the virtual servers are shared for maintaining the packet data to equalize the load imbalance factor. 


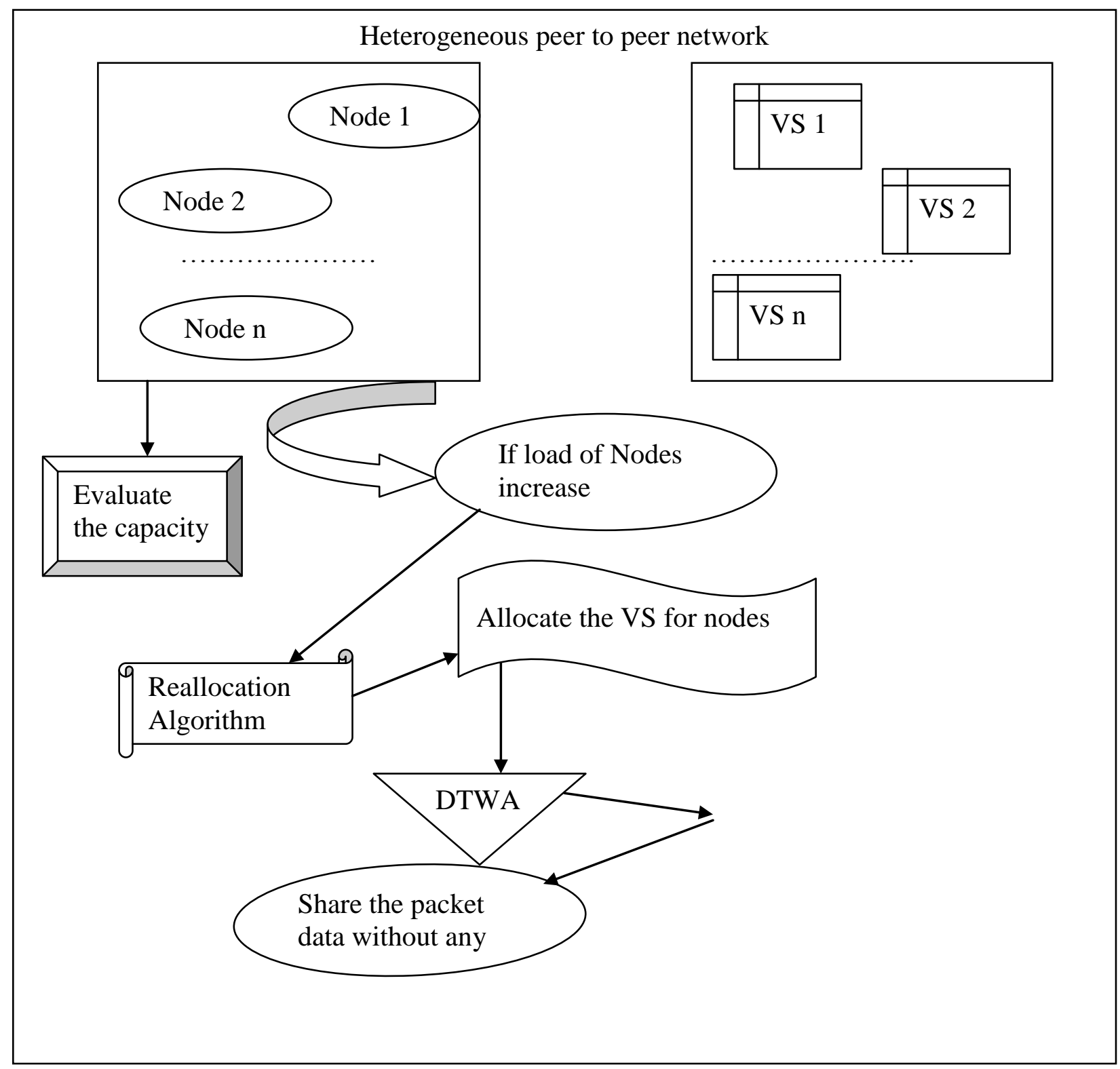

Fig. 3.1 Architecture Diagram of Time variant-peer node Heterogeneous P2P Networks using Dynamic Warping Algorithm

\section{EXPERIMENTAL EVALUATION}

The experiments were run on an Intel P-IV machine with $2 \mathrm{~GB}$ memory and $3 \mathrm{GHz}$ dual processor CPU. We are going to compare the proposed time variant peer heterogeneous P2P network using DTWA with an existing Load Balance with Imperfect Information in Structured Peerto-Peer Systems.

The performance metrics used here for the improvement of heterogeneous P2P networks are Load imbalance factor. The Load Imbalance factor for node $i \in \mathrm{N}$ is termed as

Load Imbalance Factor $=\Sigma_{\mathrm{v} \in \mathrm{i}} \mathrm{L}_{\mathrm{v}} / \mathrm{C}_{\mathrm{i}}$

For a given peer nodes, $\mathrm{N}$, and the set of virtual servers, $\mathrm{Vi}$, for each peer i $2 \mathrm{~N}$, peer I migrates a subset of its virtual servers, $\mathrm{Vi}$ (where Vi cVi), to other peers. The MS is defined as

$$
\mathrm{S}=\mathrm{U}_{\mathrm{i} \in \mathrm{N}} \mathrm{V}_{\mathrm{i}}
$$

Using these two metrics, the performance of the proposed time variant peer heterogeneous P2P network using DWA is evaluated to show the performance.

\section{RESULTS AND DISCUSSION}

When compared to an existing Load Balance with Imperfect Information in Structured Peer-to-Peer Systems, the proposed time variant peer heterogeneous P2P network using DTWA perform better in terms of load imbalance factor, movement cost, performance ratio. 


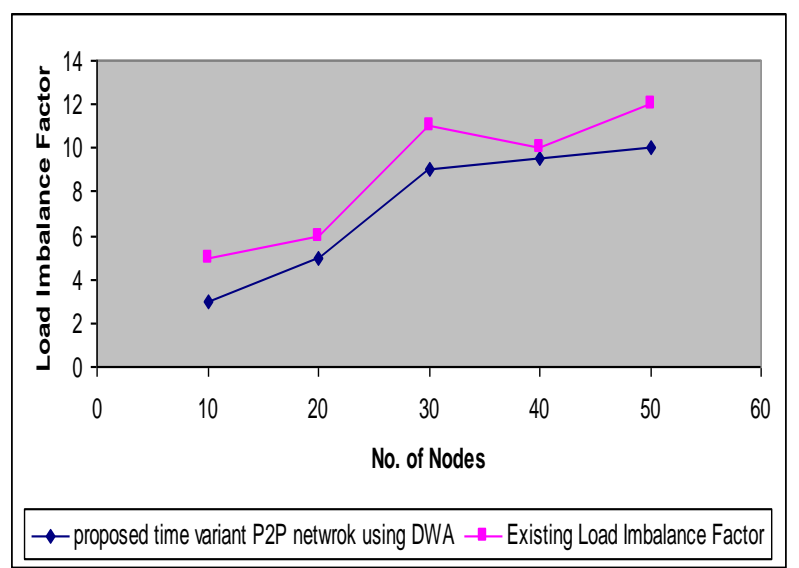

Fig 5.1 Number of nodes vs. Load imbalance Factor

Fig 5.1 describes the Load imbalance factor of nodes in the network. If the number of nodes increases, the load imbalance factor of the node remains constant at some stage in the proposed time variant peer heterogeneous $\mathrm{P} 2 \mathrm{P}$ network using DTWA. When compared to an existing Load Balance with Imperfect Information in Structured Peer-to-Peer Systems, the proposed one is good to maintain the capacity of the node.

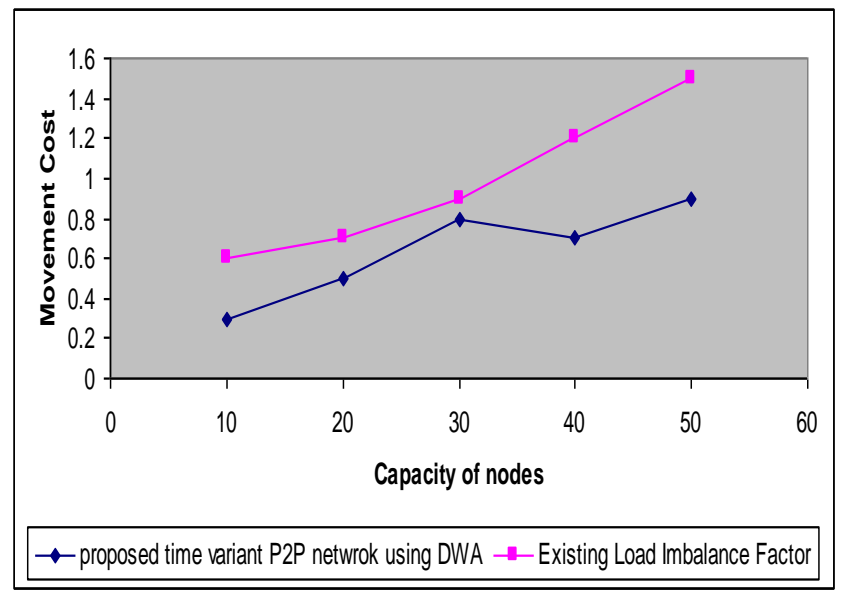

Fig 5.2 Capacity of nodes vs. Movement Cost

Fig 5.2 describes the movement cost based on the capacity of nodes in the network. If the capacity of nodes increases, the movement cost is low at some stage in the proposed time variant peer heterogeneous P2P network using DTWA. When compared to an existing Load Balance with Imperfect Information in Structured Peer-to-Peer Systems, the proposed one is good in terms of movement cost.

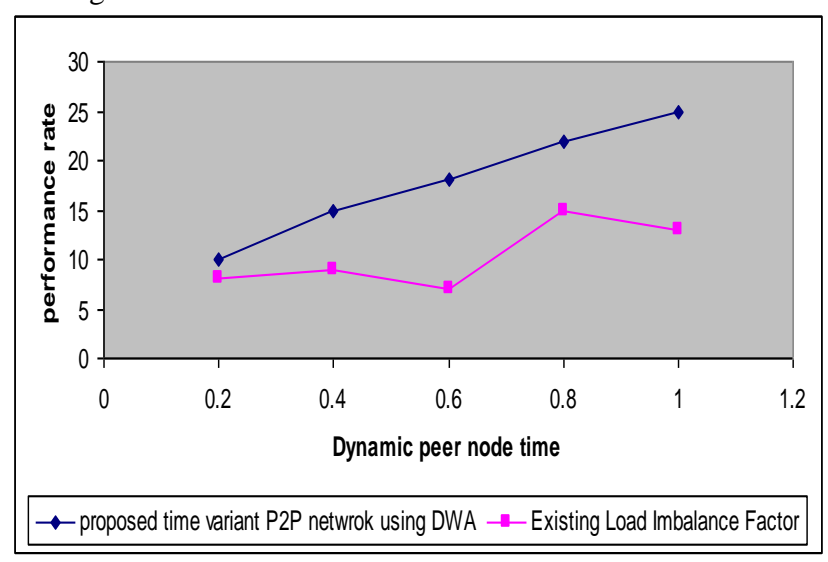

Fig 5.3 Performance rate of peer nodes
Fig 5.3 describes the performance rate of peer nodes in the network based on DW. If the dynamic peer node time increases, the performance rate of the peer node also increases in the proposed time variant peer heterogeneous P2P network using DTWA. When compared to an existing Load Balance with Imperfect Information in Structured Peer-to-Peer Systems, the proposed one performance is $20-25 \%$ high.

\section{CONCLUSION}

In this paper, we presented a time variant peer heterogeneous P2P network using DTWA with virtual severs. Our proposal is distinctive in representing the system condition with probability distributions. Not like previous solutions that frequently rely on universal knowledge of the system, each peer in our proposal separately guess the probability distributions for the facility of contributing peers and the loads of virtual servers stand on the fractional knowledge of the system. With the estimated probability distributions, each node recognizes whether it is under loaded and then reallocates its loads if it is overloaded. The simulation results shows that that the proposal performs well contrast with the existing Load Balance with Imperfect Information in Structured Peer-to-Peer Systems and outperforms solution in terms of the movement cost of virtual servers, load imbalance factor, the and/or the protocol message overhead.

\section{REFERENCES}

[1] Hung-Chang Hsiao, Hao Liao, Ssu-Ta Chen, and KuoChan Huang, "Load Balance with Imperfect Information in Structured Peer-to-Peer Systems", IEEE TRANSACTIONS ON PARALLEL AND DISTRIBUTED SYSTEMS, VOL. 22, NO. 4, APRIL 2011

[2] S. Surana, B. Godfrey, K. Lakshminarayanan, R. Karp, and I. Stoica, "Load Balancing in Dynamic Structured P2P Systems,erformance Evaluation, vol. 63, no. 6, pp. 217-240, Mar. 2006.

[3] Y. Zhu and Y. Hu, "Efficient, Proximity-Aware Load Balancing for DHT-Based P2P Systems," IEEE Trans. Parallel and Distributed Systems, vol. 16, no. 4, pp. 349361, Apr. 2005

[4] H. Shen and C.-Z. Xu, "Locality-Aware and ChurnResilient Load Balancing Algorithms in Structured P2P Networks," IEEE Trans. Parallel and Distributed Systems, vol. 18, no. 6, pp. 849-862, June 2007.

[5] C. Chen and K.-C. Tsai, "The Server Reassignment Problem for Load Balancing in Structured P2P Systems," IEEE Trans. Parallel and Distributed Systems, vol. 12, no. 2, pp. 234-246, Feb. 2008

[6] Y. Zhu, "Load Balancing in Structured P2P Networks," Handbook of Peer-to-Peer Networking, Springer, July 2009.

[7] S.M. Ross, "Markov Chains," Introduction to Probability Models, ninth ed., pp. 185-280, Academic Press, 2007.

[8] Yuzhe Tang, Shuigeng Zhou ET. AL., 'LIGHT: A QueryEfficient Yet Low-Maintenance Indexing Scheme over DHTs', IEEE TRANSACTIONS ON KNOWLEDGE AND DATA ENGINEERING, VOL. 22, NO. 1, JANUARY 2010

[9] Prashant Dewan and Partha Dasgupta, "P2P Reputation Management Using Distributed Identities and Decentralized Recommendation Chains", IEEE 
TRANSACTIONS ON KNOWLEDGE AND DATA ENGINEERING, VOL. 22, NO. 7, JULY 2010

[10] Wang Wei, Payam BarnaghiET.AL., "Probabilistic Topic Models for Learning Terminological Ontologies", IEEE TRANSACTIONS ON KNOWLEDGE AND DATA ENGINEERING, VOL. 22, NO. 7, JULY 2010

[11] D. Li, J. Cao, X. Lu, and K.C.C. Chan, "Efficient Range Query Processing in Peer-to-Peer Systems," IEEE Trans.
Knowledge and Data Eng., vol. 21, no. 1, pp. 78-91, Jan. 2009.

[12] S. Idreos, E. Liarou, and M. Koubarakis, "Continuous Multi-Way Joins over Distributed Hash Tables," Proc. Extending Data Base Technology (EDBT), 2008.

[13] G. Networks, "Groove Networks," http://www.groove.net/

products/workspace/securitypdf.gtml, 2009. 